\title{
Qualitätsmanagement bei Vertrauensgütern
}

\author{
Von Sabine Fließ
}

Vertrauensgüter sind solche Leistungen, bei denen Vertrauenseigenschaften dominieren. Vertrauenseigenschaften beziehen sich auf Leistungseigenschaften, deren Vorhandensein der Kunde auch während oder nach Inanspruchnahme der Dienstleistung nicht kostenlos beurteilen kann. Sie werfen daher bei der Qualitätsgestaltung, -steuerung und -entwicklung besondere Probleme sowohl für den Anbieter als auch für den Kunden auf. Die Zielsetzung des vorliegenden Aufsatzes besteht darin, Qualitätsstrategien bei Vertrauenseigenschaften aufzuzeigen sowie Instrumente der Qualitätsgestaltung und -steuerung vorzustellen, um diese Qualitätsstrategien umsetzen zu können. Den theoretischen Fokus bildet dabei einerseits die Informationsökonomik, andererseits der Messkostenansatz der Transaktionskostentheorie.

\section{Qualitätsprobleme bei Vertrauensgütern}

\subsection{Dienstleistungen als Vertrauensgüter}

Im Rahmen der Informationsökonomik werden Such-, Erfahrungs- und Vertrauenseigenschaften unterschieden (Nelson 1970; Darby/Karni 1973): „Search qualities, which are known before purchase, experience qualities which are known costlessly only after purchase, and credence qualities which are expensive to judge even after purchase" (Darby/Karni 1973, S. 69). Alle Leistungen weisen Such-, Erfahrungs- und Vertrauenseigenschaften in

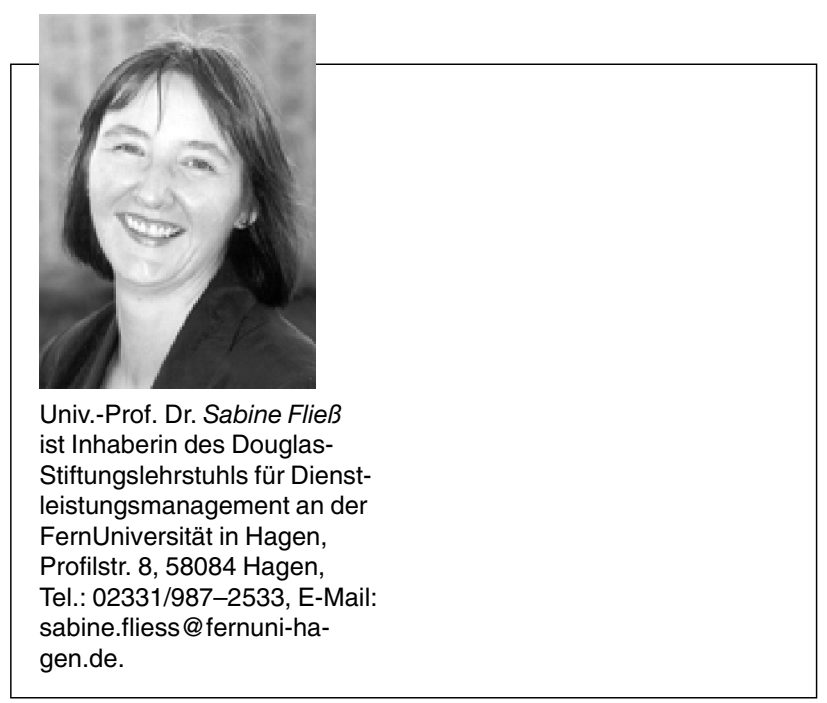

unterschiedlichem Maße auf (Weiber/Adler 1995a; 1995b; Kaas/Busch 1996), wobei nach der Dominanz der jeweiligen Eigenschaften Such-, Erfahrungs- und Vertrauensgüter unterschieden werden (Schade/Schott 1993, S. 18).

Dienstleistungen sind dadurch gekennzeichnet, dass sie der Integration externer Faktoren bedürfen. Als externe Faktoren werden dabei die Faktoren bezeichnet, die Eigentum des Kunden sind und lediglich zeitlich begrenzt in den Verfügungsbereich des Anbieters gelangen, wie etwa Personen, Objekte, Tiere und Pflanzen, Nominalgüter, Rechte und Informationen (Engelhardt/Kleinaltenkamp/Reckenfelderbäumer 1993, S. 401). Aufgrund der Notwendigkeit der Integration externer Faktoren stellen Dienstleistungen im Gegensatz zu Austauschgütern ( $\mathrm{Al}$ chian/Woodward 1988), die zum Zeitpunkt des Verkaufs bereits existieren, Leistungsversprechen dar (Kaas 1992a; Schade/Schott 1993, S. 17). Diese können in standardisierte und nicht standardisierte Leistungsversprechen unterteilt werden (Schade/Schott 1993, S. 17; Woratschek 1996). Bei standardisierten Leistungsversprechen dominieren Erfahrungseigenschaften, während bei nicht standardisierten Leistungsversprechen und sog. Kontraktgütern (Schade/Schott 1993; Kaas 1992a) Vertrauenseigenschaften vorherrschen (Woratschek 1996, S. 63). Beispiele hierfür sind Unternehmensberatungsleistungen, Rechtsberatungen in komplexeren Fällen oder Wirtschaftsprüfungsleistungen.

Legt man die drei Leistungsdimensionen einer Dienstleistung - Leistungspotenzial, Leistungserstellungsprozess und Leistungsergebnis - zugrunde (Corsten 1997, S. 119 ff.; Engelhardt/Kleinaltenkamp/Reckenfelderbäumer 1993, S. 398; Hilke 1989; Meffert/Bruhn 2003, S. 27 ff.), so werden Vertrauenseigenschaften meist mit dem Leistungsergebnis in Verbindung gebracht. Sie können aber auch - wie Adler mit den ,veiled characteristics" bezogen auf die Qualifikation und Fähigkeit sowie dem ,veiled effort" bezogen auf die Anstrengung und den Leistungswillen verdeutlicht (Adler 1996, S. 64) im Leistungserstellungsprozess und im Leistungspotenzial auftreten. Vertrauenseigenschaften in Verbindung mit der Notwendigkeit, externe Faktoren in den Leistungserstellungsprozess zu integrieren, werfen besondere Qualitätsprobleme auf.

\subsection{Qualitätsbeurteilungsprobleme des Nachfragers}

Qualität wird nach DIN 55350 Teil 11 definiert als „Gesamtheit von Eigenschaften und Merkmalen eines Produktes oder einer Tätigkeit, die sich auf deren Eignung zur Erfüllung gegebener Erfordernisse bezieht. Die Er- 
fordernisse ergeben sich aus dem Verwendungszweck des Produktes oder dem Ziel der Tätigkeit unter Berücksichtigung der Realisierungsmöglichkeiten." Welche Leistungsmerkmale dem Kunden welchen Nutzen stiften, hängt vom jeweiligen Kunden ab. In der mikroökonomischen Theorie, auf der auch die Informationsökonomik basiert, wird der Nutzen durch die i. d. R. exogen gegebenen Präferenzen des Nachfragers bestimmt. Der verhaltenswissenschaftlich geprägten Marketingliteratur zufolge ergibt er sich aus den Bedürfnissen, Motiven und Präferenzen des Konsumenten (Business-to-ConsumerMarketing) oder der Nutzungskonzeption und dem zu lösenden Problem des nachfragenden Unternehmens (Business-to-Business-Marketing). Übereinstimmung besteht zwischen verhaltenswissenschaftlichen und mikroökonomischen Ansätzen dahingehend, dass die Qualität einer Dienstleistung vom Nachfrager bestimmt wird. In Verbindung mit der Sichtweise der Dienstleistung als Eigenschaftsbündel, wobei jede Eigenschaft einen spezifischen Nutzen stiftet (Kaldor 1950/51; Lancaster 1966), ergibt sich die Qualitätsbewertung als Gegenüberstellung von Soll- und Ist-Eigenschaften der Dienstleistung (Gössinger 2003, S. 36 und die dort angegebene Literatur).

Unterstellt man nutzenmaximierendes Verhalten, so ist der Nachfrager an der Beurteilung aller für ihn relevanten nutzenstiftenden Eigenschaften interessiert. Bei Vertrauensgütern kann der Nachfrager jedoch nicht oder nur zu sehr hohen Kosten zwischen Anbietern guter und Anbietern schlechter Qualität differenzieren. Dies eröffnet dem Anbieter einen Verhaltensspielraum, den er opportunistisch ausnutzen kann. So kann ein Wirtschaftsprüfer etwa behaupten, eine sorgfältige Prüfung vorgenommen zu haben, obwohl dies letztlich nicht zutrifft. Da die Sorgfalt der Prüfung für den Kunden eine Vertrauenseigenschaft darstellt, läuft er Gefahr, schlechte Qualität zu einem überhöhten Preis zu erwerben. Fehlende Beurteilungsmöglichkeiten von Vertrauenseigenschaften können dann zum Kaufverzicht bzw. sogar zu Marktversagen führen (siehe Kaas 1992b für das Beispiel umweltfreundlicher Produkte).

Verschärft wird das Qualitätsbeurteilungsproblem des Nachfragers dadurch, dass der Verlauf des Leistungserstellungsprozesses und die Qualität des Leistungsergebnisses aufgrund der Integration externer Faktoren auch von der Qualität dieser abhängt (Brentani/Ragot 1996; Fließ 2001, S. 72 ff.; Kelley/Skinner/Donnelly 1992; Meyer/Westerbarkey 1995; Schneider/Bowen 1983, S. 95). Erschwerend wirkt sich hierbei aus, dass der Nachfrager - trotz seiner Funktion als Co-Produzent die Verantwortung für die Qualität des Leistungsergebnisses häufig beim Anbieter sieht (Ennew/Binks 1999).

\subsection{Qualitätsmanagementprobleme des Anbieters}

Ähnlich wie der Nachfrager sieht sich auch der Anbieter Qualitätsbeurteilungsproblemen des externen Faktors gegenüber, da nicht grundsätzlich davon auszugehen ist, dass der Nachfrager immer die höchste Qualität liefert, sei es aus Unkenntnis oder Unfähigkeit (Fähigkeitsbarrieren) oder sei es mit Absicht (Willensbarrieren) (Fließ 1996, S. 93 f.; Witte 1973, S. 6 f.). So können bei einer Unternehmensberatungsleistung etwa Mitarbeiter, die befürchten, dass ihr Arbeitsplatz wegrationalisiert wird, dem Berater Informationen vorenthalten, verfälschen oder einfach die Lieferung verzögern. Da die Strategieempfehlung auf der Einbeziehung der entsprechenden Informationen beruht, wirkt sich diese Schlechtlieferung qualitätsmindernd auf das Leistungsergebnis aus. Externe Faktoren weisen somit ebenfalls Such-, Erfahrungs- und Vertrauenseigenschaften auf (Helm 1997; Helm/Kuhl 1997; Hempe 1997, S. 33 f.). Dies eröffnet dem Nachfrager opportunistisch zu nutzende Verhaltensspielräume und kann zu den aus der PrincipalAgent-Theorie bekannten Problemen von hidden characteristics, hidden intention und hidden action führen (Arrow 1985; Kaas 1991; 1992a; Kleinaltenkamp 1992; Ross 1973; Spremann 1988).

Eine besondere Rolle kommt im Rahmen des Qualitätsmanagements den steuernden externen Prozessinformationen des Nachfragers zu. Externe Prozessinformationen sind Informationen, die der Nachfrager während eines Leistungserstellungsprozesses zur Verfügung stellt (Kleinaltenkamp 1993, S. 107; Kleinaltenkamp/Haase 1999, S. 174). Steuernde Prozessinformationen wirken im Sinne des dispositiven Faktors (Gutenberg 1983) auf die Kombination der Elementarfaktoren ein (Gersch 1998, S. 198; Kleinaltenkamp/Marra 1997, S. 64; Kleinaltenkamp/Haase 1999, S. 174), z. B. die aus den Kundenzielen abgeleiteten Vorstellungen über die termingerechte Fertigstellung einer Reparatur (Krimm 1995, S. 15 ff.).

Steuernde externe Prozessinformationen führen dazu, dass der Anbieter seine alleinigen Verfügungsrechte bezüglich des Einsatzes und der Kombination der Faktoren einbüßt (Kleinaltenkamp 1997, S. 90). Aufgrund der daraus resultierenden integrativen Disposition ist auch die Qualitätsgestaltung und -steuerung des Anbieters eingeschränkt. Der Anbieter sieht sich somit einem Qualitätsbeurteilungsproblem hinsichtlich der Qualität der externen Faktoren und einem Qualitätsmanagementproblem hinsichtlich des Leistungserstellungsprozesses gegenüber.

Das Qualitätsbeurteilungsproblem des Nachfragers wird in der bisherigen informationsökonomisch begründeten Marketingliteratur vor allem als ein Problem des Signaling und der Nutzung von Institutionen (z. B. Garantien, Reputation) im Vorfeld der Leistungserbringung angesehen. Lösungen werden somit im Bereich der Kommunikationspolitik des Anbieters bzw. der Leistungsbegründung (Kaas 1990, S. 544), nicht aber im Bereich des Qualitätsmanagements bzw. der Leistungsfindung (Kaas 1990, S. 543) des Anbieters gesehen.

Zur Vermeidung oder Verringerung von durch externe Faktoren bedingten Qualitätsschwankungen auf Anbieterseite werden vor allem Marktsegmentierungs-, Standardisierungs- und Automatisierungsmaßnahmen vorgeschlagen (Corsten 1996; Gersch 1995, S. 36 ff.; Meyer 1987; 
Schnittka 1998, S. 80 ff.). Diese Maßnahmen setzen während der Leistungserbringung an, sind allerdings auf Vertrauensgüter nur begrenzt anwendbar. Keine der vorgeschlagenen Maßnahmen bezieht sich direkt auf das Qualitätsmanagement von Dienstleistungen, bei denen Vertrauenseigenschaften dominieren. Daher sollen im Folgenden Qualitätsstrategien vorgestellt werden, die an den Ursachen der Existenz von Such-, Erfahrungs- und Vertrauenseigenschaften ansetzen - den Kosten der Qualitätsmessung.

\section{Messkosten als Ursache von Such-, Erfah- rungs- und Vertrauenseigenschaften}

Der Messkostenansatz (Williamson 1996, S. 65) wurde durch die Washingtoner Schule geprägt (North 1990, Fußnote 1, S. 27 f.; Barzel 1982; 1985; siehe auch Alchian/Demsetz 1972; Cheung 1969; Hallwood 1990; McManus 1975). Den Ausgangspunkt bildet die Überlegung, dass jede Leistung - gemeint sind hier sowohl Produkte und Dienstleistungen als auch Ressourcen wie menschliche Arbeitsleistung, Sachanlagen oder Grundstücke - als Bündel verschiedener Eigenschaften interpretiert werden kann, an deren Verfügungsrechten der Transaktionspartner interessiert ist. Jeder Austausch zwischen Anbieter und Nachfrager beinhaltet eine Messung der zu tauschenden Eigenschaften (Barzel 1982, S. 32; McManus 1975, S. 337; North 1992, S. 35); er beinhaltet des Weiteren eine - zumindest implizite - Einigung über die dabei zugrunde gelegte Messmethode (McManus 1975, S. 337). Dies ist deshalb erforderlich, weil der Preis des Gutes an seine Eigenschaften gekoppelt ist, d. h. der Käufer will nur den Preis zahlen, der dem Wert der Eigenschaften entspricht. Dies setzt die Messung der Eigenschaften voraus.

Wird der Begriff der „Messung” weit ausgelegt, so ist jede Eigenschaftsprüfung und damit jede Qualitätsbeurteilung Gegenstand einer solchen Messung. Die Bandbreite reicht von technischen Messverfahren bis hin zur subjektiven Beurteilung, etwa wenn eine Leistung lediglich in Augenschein genommen wird. Die Eigenschaftsmessung einer Leistung ist mit Messkosten verbunden (Barzel 1982, S. 27), die in drei Klassen unterteilt werden können (Fließ 2001, S. 182 ff.):

1. Direkte Messkosten: Kosten der Ermittlung der Eigenschaftsverteilung der Leistungen;

2. Messfehlerkosten: Opportunitätskosten, die entstehen, weil Messfehler zu Nutzenentgang führen, wobei die Höhe der Messfehlerkosten nur dann ermittelt werden kann, wenn die „wahre“ Eigenschaftsverteilung bekannt ist;

3. Opportunismuskosten: Opportunitätskosten, die entstehen, weil Messfehler opportunistisch ausgenutzt werden, das sog. ,cheating“-Problem [1].

Bei Vertrauensgütern besteht das Hauptproblem darin, dass die direkten Messkosten bezüglich der Feststellung von Vertrauenseigenschaften prohibitiv hoch sind. Beispielsweise kann der Patient nach einer Blinddarmoperation - sofern kein akuter Blinddarm vorlag - nicht ohne hohe direkte Messkosten feststellen, ob der Blinddarm überhaupt entfernt wurde, da er sich nach der Rekonvaleszenz i. d. R. nicht anders fühlt als vorher. Daher wird häufig auf Indikatoren (Spence 1974, S. 7) oder sog. leistungsübergreifende Informationssubstitute (Adler 1996) zurückgegriffen. Die Höhe der Messfehlerkosten wird direkt durch die Qualität des Indikators beeinflusst. Als Indikatoren können etwa die Kompetenz des Dienstleisters (Adler 1996, S. 164 f.), seine Kapazitätsauslastung (Emons 2001, S. 377 ff.) oder seine Reputation herangezogen werden (Kaas 1992a, S. 894 f.; Spremann 1988). Je höher die Messfehlerkosten sind, desto eher eröffnen sich opportunistisch zu nutzende Verhaltensspielräume, die die Opportunismuskosten in Form eines entgangenen Nutzens (der Kunde zahlt mehr als es dem Wert des Gutes entspricht) erhöhen. Zwischen den Kostenarten besteht somit ein Trade-off: Direkte Messkosten sinken, wenn eine ungenauere Messmethode verwendet wird, die die Messfehlerkosten erhöht. Eine Methode mit einem größeren Messfehler erhöht bei Opportunismusneigung die Opportunismuskosten.

Im Falle von Vertrauenseigenschaften sind Messkosten prohibitiv hoch. Prohibitiv hohe Messkosten entstehen, wenn keine technischen Methoden der Eigenschaftsmessung zur Verfügung stehen, die Kosten der Messung den Wert der zu tauschenden Leistungen übersteigen oder die Opportunitätskosten des Ressourceneinsatzes der Messungen so hoch sind, dass die Alternativverwendung zu einem höheren Nutzen führt als der Tausch der zu messenden Leistungen. Dies ist in den folgenden Fällen gegeben:

- Der Nachfrager kann seinen Bedarf an einer Leistung nicht einschätzen (Darby/Karni 1973; Mills 1990; Emons 2001; Wolinsky 1995, S. 117); es liegt somit mangelnde Problemevidenz vor (Malicha 2000). So kann er etwa nicht feststellen, ob er zur Regelung eines Rechtsstreits wirklich einen Rechtsanwalt benötigt, ob sein Pkw tatsächlich repariert werden muss, der Blinddarm oder die Mandeln zu entfernen sind u. Ä. Dies gilt u. U. auch für den Anbieter, etwa den Unternehmensberater, der nicht weiß, welche Informationen er benötigt, um das Problem des Kunden zu spezifizieren.

- Ein Gut wird zusammen mit anderen Gütern, deren Eigenschaften unsicher sind, genutzt, so dass der Output nicht eindeutig einem Gut zugeordnet werden kann, z. B. Waschmaschine mit Reparaturleistung (Darby/Karni 1973).

- Leistungsergebnisse sind hochkomplex, so dass ihre Nutzenmerkmale kaum einzeln $\mathrm{zu}$ beurteilen sind (Weißenberger 1998, S. 618).

- Die Bedarfserfüllung, d. h. der Produktions- oder Leistungserstellungsprozess ist zumindest in einem subjektiven Sinne stochastisch, d. h. zufallsabhängig (Darby/Karni 1973; Mills 1990, S. 35). So hängt beispielsweise der Erfolg eines Handelsvertreters zum 
einen von seinem Verhalten (Sorgfalt, Fleiß) ab, aber auch von externen Umständen (Größe des Verkaufsgebietes, Glück u. Ä.), so dass etwa bei einem erfolgreichen Handelsvertreter nicht klar ist, ob sein Erfolg auf Glück oder auf Anstrengung zurückzuführen ist (Mills 1990). Dies gilt vice versa für den Anbieter, dessen Leistungserstellungsprozess in starkem Maße von den externen Faktoren des Nachfragers abhängt.

- Aufgrund häufiger Änderungen, großer Variationen der Leistungsmerkmale sowie der Einzigartigkeit einer Leistung kann ein Nachfrager keinen eindeutigen Zusammenhang zwischen Input, dem Produktions- oder Leistungserstellungsprozess und dem Leistungsergebnis erkennen (Cheung 1983, S. 7; Mills 1990, S. 36; Ouchi 1980, S. 130 f.).

Das Auftreten von Vertrauenseigenschaften ist dabei in starkem Maße vom jeweiligen Wissen der Beteiligten abhängig (Adler 1996, S. 134) und kann bezüglich der zu messenden Eigenschaften (Messgegenstand), des Messzeitpunktes oder der Messmethode variieren. In diesem Sinne ist auch die Tatsache zu interpretieren, dass etwa Arztkindern wesentlich seltener die Mandeln herausgenommen werden als Kindern, deren Eltern keine Ärzte sind (Emons 2001, S. 376).

Direkte Messkosten, Messfehlerkosten und Opportunismuskosten können durch die Einigung darüber, wer die Messung $\mathrm{zu}$ welchen Zeitpunkten an welchen Gegenständen mit welchem Messverfahren durchführt, gesenkt werden. Hierzu zählen auch Transaktionsarrangements und Institutionen, wie Marken, Garantien und Reputation (Barzel 1985), die der Absicherung und Durchsetzung der Messung dienen (Cheung 1969, S. 24; North 1990, S. 27). Hierdurch werden die Messmethode, der Messzeitpunkt, der Messgegenstand und der die Messung Durchführende bestimmt. Allerdings sind mit der Etablierung, Nutzung und Durchsetzung dieser Arrangements und Institutionen ebenfalls Kosten verbunden. Werden direkte Messkosten, Messfehlerkosten und Opportunismuskosten als Messkosten erster Ordnung bezeichnet, so handelt es sich bei den Kosten der Vereinbarung und Absicherung der Messung um Messkosten zweiter Ordnung (Fließ 2001, S. 190 ff.).

Qualitätsstrategien als Alternative zur Nutzung von Marktinstitutionen im Vorfeld der Leistungserbringung müssen an den Ursachen der prohibitiv hohen Messkosten ansetzen.

\section{Qualitätsstrategien im Rahmen des Quali- tätsmanagements}

\subsection{Aufgaben des Qualitätsmanagements}

Als zentrale Aufgaben des Qualitätsmanagements lassen sich die Qualitätsgestaltung, die Qualitätssteuerung und die Qualitätsentwicklung herausstellen (siehe zu anderen Unterteilungen Bruhn 2001; Bruhn/Stauss 2000). Im Rahmen der Qualitätsgestaltung von Vertrauensgütern kommt es vor dem Hintergrund der Qualitätsbeurteilungsprobleme von Anbieter und Nachfrager darauf an, Qualitätsmerkmale möglichst nachprüfbar zu gestalten. Zielsetzung ist die Verringerung der prohibitiv hohen Messkosten.

Aufgabe der Qualitätssteuerung ist die Qualitätssicherung, indem aufgrund von Qualitätsmessungen Abweichungen ermittelt werden, die es mittels geeigneter Maßnahmen zu beseitigen gilt. Zielsetzung ist die Aufrechterhaltung eines einheitlichen Qualitätsniveaus mittels geeigneter Qualitätssicherungsinstrumente. Dies stellt angesichts möglicher Qualitätsschwankungen externer Faktoren durchaus eine Herausforderung dar.

Die Qualitätsentwicklung bezieht sich sowohl auf die Qualitätsgestaltung als auch auf die Qualitätssteuerung. Im Rahmen der Qualitätsgestaltung hat der Anbieter zum einen der Veränderung der Qualitätsdimensionen und -merkmale als Konsequenz der Veränderung von Nutzenmerkmalen des Kunden und der Qualitätsänderungen der Wettbewerber Rechnung zu tragen. Im Rahmen einer zukunftsweisenden Qualitätssteuerung sind Strukturen zu schaffen und Instrumente zu implementieren, die es erlauben, Veränderungen von Qualitätsdimensionen, -merkmalen und -standards rechtzeitig zu erkennen und qualitätsverbessernde Maßnahmen zu ergreifen.

Im Folgenden sollen verschiedene Strategien zur Qualitätsgestaltung, Qualitätssteuerung und Qualitätsentwicklung dargestellt werden.

\subsection{Strategien und Instrumente zur Qualitätsge- staltung}

\subsubsection{Debundlingstrategien zur Senkung von Mess-} kosten

Unter Bundling oder Bündelung wird im Allgemeinen das ,kombinierte Angebot von zwei oder mehr Gütern als Paket zu einem Gesamtpreis“ (Priemer 2000, S. 31) verstanden. Hierbei kann es sich um die Kombination von Produkten mit Dienstleistungen handeln, z. B. Maschine mit Wartungsvertrag, von Produkten mit anderen Produkten, z. B. Computerpakete bestehend aus Bildschirm, Zentraleinheit, Tastatur, Maus u. Ä., oder von mehreren Dienstleistungen, z. B. Problemanalyse in Verbindung mit einer Strategieberatung bei einem Unternehmensberater.

Aus messkostentheoretischen Aspekten ist dabei insbesondere die Bündelung als physische Zusammenfassung von Einzelleistungen von Interesse, nicht jedoch die unter absatzpolitischen, insbesondere preispolitischen Aspekten erfolgende gedankliche Zusammenfassung von weiterhin isoliert bestehenden Einzelleistungen zu Leistungspaketen (Olderog/Skiera 2000; Simon 1994, S. 729; Yadav/Monroe 1993). Werden mehrere Einzelleistungen zu einem physisch nicht mehr in Einzelleistungen aufspaltbaren Gesamtpaket zusammengefügt, so entsteht dadurch eine neue Leistung mit anderen Nutzen- und damit auch Qualitätsmerkmalen - ein Problem, das aus 
messkostentheoretischer Perspektive mit dem Problem der Messung von Einzelbeiträgen bei Teamproduktion vergleichbar ist (Alchian/Demsetz 1972, S. 779). Hieraus resultiert eine Veränderung der Messmöglichkeiten des Nachfragers. Geht man von den beiden grundsätzlichen Möglichkeiten der Input- und der Outputmessung aus, so wird eine vorherige Inputmessung in eine Outputmessung überführt. Eine solche Outputmessung ist aber aufgrund des Vertrauensgutcharakters mit prohibitiv hohen Messkosten verbunden.

Legt man nur die Messkosten erster Ordnung zugrunde, so ist ein Anbieter von Vertrauensleistungen, der sich im Qualitätswettbewerb von seinen Wettbewerbern durch das Signalisieren höherer Qualität abheben möchte, gut beraten, eine Strategie des Debundling zu verfolgen. So können etwa bei einer Maschinenreparatur Diagnose und Reparatur als voneinander getrennte Leistungen angeboten werden, um zu verhindern, dass bei Unterauslastung der Kapazitäten des Anbieters Wartungs- und Reparaturleistungen angeboten werden, deren Notwendigkeit der Nachfrager aufgrund der Vertrauenseigenschaft der Reparaturanfälligkeit nicht beurteilen kann (Darby/Karni 1973; Emons 2001, S. 375). Zu finden ist eine solche Debundlingstrategie beispielsweise im Gesundheitsbereich, wo die Diagnose vom Hausarzt vorgenommen wird, der dann zwecks Weiterbehandlung an einen Spezialisten überweist [2].
Die Debundlingstrategie kann von Leistungsergebnissen auch auf Leistungserstellungsprozesse übertragen werden. So lassen sich viele Leistungserstellungsprozesse in voneinander isolierbare Teilprozesse zerlegen, was einem prozessbezogenen Debundling entspricht. Werden nun zwischen den isolierbaren Teilprozessen Messzeitpunkte eingeführt, so kommt es zu einer besseren Überprüfbarkeit der vorhandenen Qualitätseigenschaften. Damit steigt die Genauigkeit der Messung (geringere Messfehler) bei gleichzeitig höheren direkten Messkosten (häufigere Messung), was aber letztlich die Opportunismuskosten verringert (Opportunismusneigung wird früher aufgedeckt). Durch die Einführung von Messpunkten werden Vertrauenseigenschaften in Erfahrungseigenschaften überführt.

Als Methode für die Zerlegung des Leistungserstellungsprozesses kann das ServiceBlueprint verwendet werden. In einem ServiceBlueprint werden auf der horizontalen Ebene die einzelnen Aktivitäten in einem Leistungserstellungsprozess chronologisch dargestellt, während fünf vertikale Linien diese Aktivitäten nach ihrer „Entfernung" vom Kunden strukturieren (siehe zu verschiedenen Versionen den Überblick bei Kleinaltenkamp 2000). Die Line of Interaction trennt dabei die Kundenaktivitäten von den Anbieterinteraktivitäten, die Line of Visibility trennt die für den Kunden sichtbaren Anbieteraktivitäten von solchen, die für den Kunden nicht sichtbar sind,

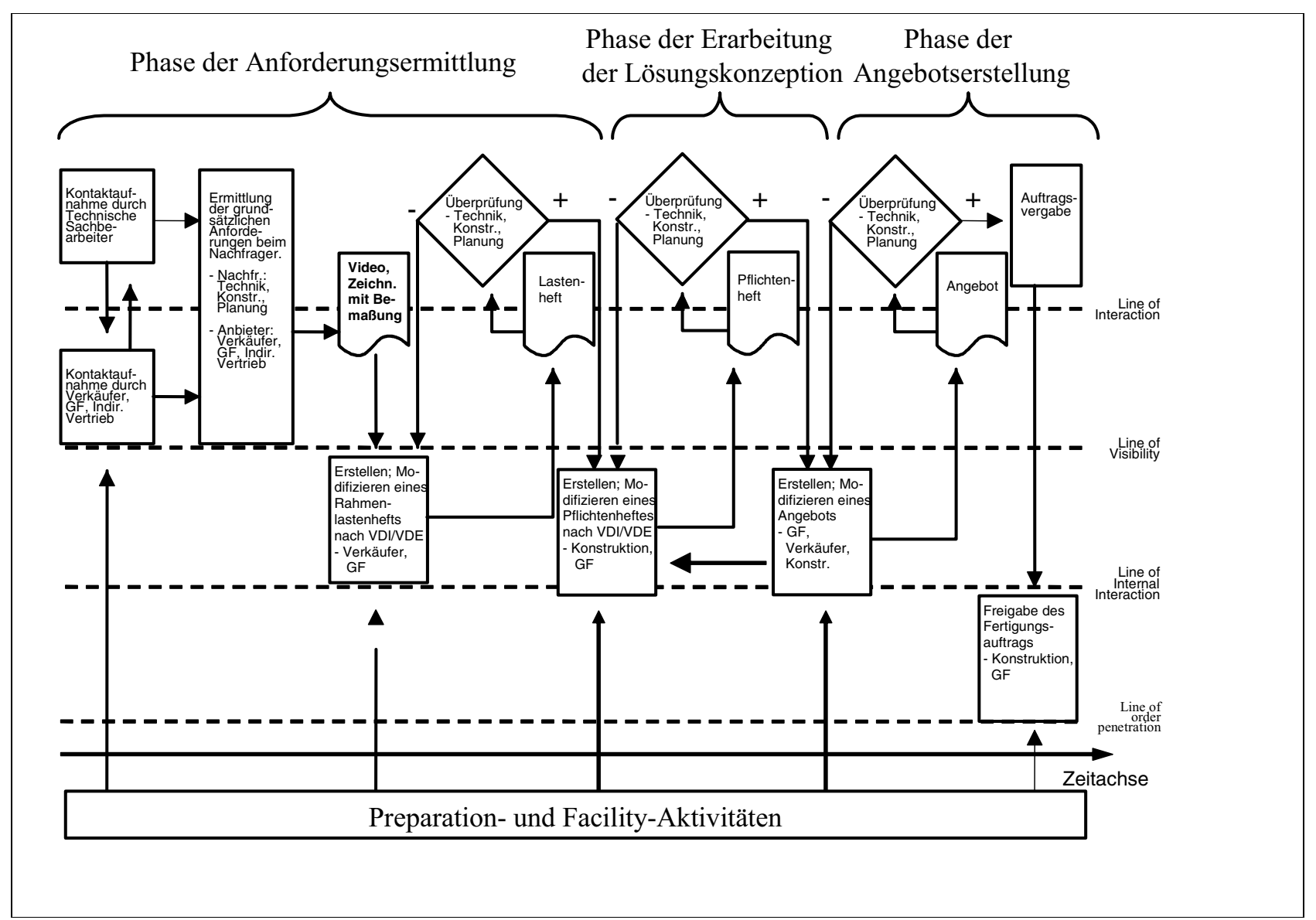

Abb. 1: Beispiel eines Akquisitionsprozesses für einen Kundenintegrationsprozess mit hohem Integrationsgrad (Quelle: In Anlehnung an Weiber/Jacob 2000, S. 583) 


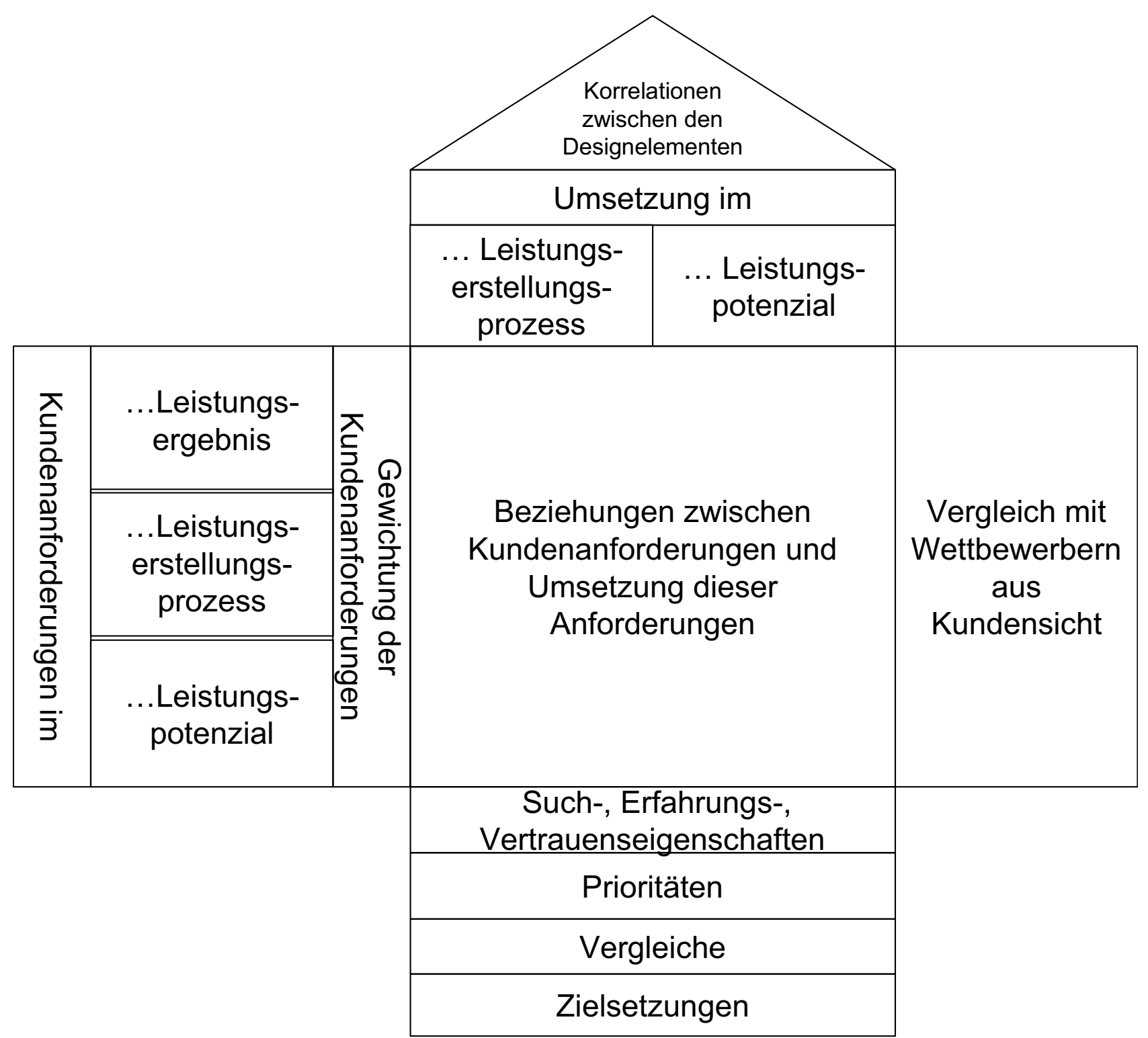

Abb. 2: Informationsökonomische und dienstleistungsspezifische Aspekte im House of Quality

die Line of Internal Interaction trennt Aktivitäten des Kundenkontaktpersonals von solchen anderer Personen, Abteilungen oder Stellen. Die Line of Order Penetration trennt durch den Kunden angestoßene Aktivitäten (kundeninduzierte) von solchen Aktivitäten, die vom Kunden unabhängig sind (autonome Aktivitäten des Anbieters), während die Line of Implementation die kundenunabhängigen Aktivitäten in Vorbereitungsaktivitäten und Beschaffungsaktivitäten unterteilt (Fließ 2001, S. 44 ff.).

Abb. 1 zeigt das Blueprint eines Akquisitionsprozesses, wobei hier die autonomen Aktivitäten des Anbieters nicht weiter unterteilt werden. Es enthält drei Mess- und Absicherungspunkte, die sich jeweils an der Line of Interaction befinden und den Leistungserstellungsprozess in drei Phasen unterteilen. Der erste Mess- und Absicherungspunkt umfasst das Lastenheft und schließt die Phase der Anforderungsermittelung des Nachfragers ab. Die zweite Phase umfasst die Erarbeitung der Lösungskonzeption und wird durch das Pflichtenheft abgeschlossen.
Die dritte Phase schließlich beinhaltet die Angebotserstellung und wird durch das Angebot abgeschlossen (Fließ 2001, S. 194 ff.).

Debundlingstrategien überführen durch die Etablierung neuer Messzeitpunkte Vertrauenseigenschaften in Erfahrungseigenschaften, auch wenn kaum alle Vertrauenseigenschaften in Erfahrungseigenschaften umgewandelt werden können, und ermöglicht die Nutzung bisher nicht genutzter Institutionen, z. B. Garantien.

Während Debundlingstrategien durch die Einführung neuer Messzeitpunkte Vertrauens- in Erfahrungseigenschaften überführen, setzt die Visualisierungsstrategie direkt am Messgegenstand an.

\subsubsection{Visualisierungsstrategien zur Senkung von Messkosten}

Leistungen strahlen, wie Ellinger anhand der Informationsfunktion des Produktes herausstellte, Informationen 
über sich selbst in die Marktsphäre aus (Ellinger 1966). Hierzu zählen neben Existenz- und Herkunftsinformationen auch Qualitätsinformationen, die sich in evidente (erkennbare) und latente (nicht erkennbar, verborgene) Informationen unterscheiden lassen. Latente Informationen beruhen auf funktions- oder dispositionsbedingter Latenz, wobei funktionsbedingte Latenz auf der im Produktplan konstruktiv festgelegten Grundfunktion beruht und somit von der Produktionstechnologie abhängt. Dispositionsbedingte Latenz liegt dann vor, wenn die Informationsausstrahlung zwar technologisch realisierbar wäre, aber aus Vermarktungsüberlegungen des Anbieters auf sie verzichtet wird (Ellinger 1966, S. 266). Latente Produktinformationen verursachen naturgemäß höhere Messkosten als evidente Produktinformationen.

Ein Anbieter von Vertrauensgütern überlegener Qualität kann somit durch Veränderung der Produktionstechnologie latente in evidente Produktinformationen umwandeln. Die Visualisierung kann dabei so erfolgen, dass der Nachfrager selbst Messungen vornehmen kann oder dass ihm Messungen erleichtert werden, d. h. zu niedrigeren Messkosten durchführbar sind.

Visualisierungen entsprechen - je nach eingesetzter Technologie - der Umwandlung von Vertrauenseigenschaften in Erfahrungs- oder Sucheigenschaften. Grundlage für die Planung kann das House of Quality als Instrument des Quality Function Deployment sein (Akao 1990; Eringa/Boer 1998; Saatweber 1994; Stuart/Tax 1996; siehe $A b b$. 2). Hierbei werden die Kundenanforderungen nach Anforderungen an das Leistungsergebnis, an den Leistungserstellungsprozess und an das Leistungspotenzial gruppiert. Die Umsetzung in Designmerkmale kann ebenfalls in Designelemente im Leistungspotenzial und Designelemente im Leistungserstellungsprozess strukturiert werden. Designelemente des Leistungsergebnisses ergeben sich aus den Elementen im Leistungserstellungsprozess und im Leistungspotenzial, so dass hierfür keine gesonderte Kategorie gebildet werden muss.

Die Designelemente können dann in einer Zeile unterhalb der Beziehungsmatrix in Such-, Erfahrungs- und Vertrauenseigenschaften klassifiziert werden. In Kombination mit den Benchmarking-Ergebnissen können hier unter wettbewerbs- und messtheoretischen Aspekten qualitätsverbessernde Möglichkeiten der Dienstleistungsgestaltung aufgedeckt werden. Unter Rückgriff auf den morphologischen Kasten lassen sich auch neue Realisationsmöglichkeiten entwickeln.

Gelingt die Visualisierung für das Leistungsergebnis, so werden Messungen am Output möglich, andernfalls handelt es sich um Messungen am Input oder im Leistungserstellungsprozess zu niedrigeren Messkosten als bisher. Bei einem Restaurant ist die Küche i. d. R. verborgen, so dass der ,normale“ Restaurantbesucher nicht feststellen kann, ob frische oder gefrorene Zutaten verwendet oder ob sogar Halbfertigfabrikate wie geschälte Kartoffeln oder bereits panierte und konfektionierte Hähnchenbrüs- te verwendet werden (Experten unter den Gästen können dies u. U. am Geschmack feststellen). Die Öffnung der Küche, die Integration der Küche in das Restaurant, die Zubereitung am Tisch des Gastes u. Ä. verschieben die „Line of Visibility“, so dass der Gast nun selbst in der Lage ist, die Zubereitung der Speisen zu verfolgen und die Qualität zu beurteilen.

Die Visualisierung muss nicht notwendigerweise dazu führen, dass der Kunde selbst das Vorhandensein von Qualitätseigenschaften feststellen kann, sondern sie kann sich auch auf die Dokumentation von Messungen des Anbieters beziehen, so dass der Nachfrager auf eigene Messungen verzichten kann und den Messungen des Anbieters vertraut. Ein Wirtschaftsprüfer, der seine Prüfungsplanung und seine Prüfungsergebnisse dokumentiert, versetzt den Nachfrager bei Bedarf in die Lage, seine Leistung zu überprüfen. Latente Informationen, d. h. die im Kopf des Wirtschaftsprüfers bzw. seines Prüfungsleiters und seiner Mitarbeiter vorhandene Vorgehensweise, werden in evidente Informationen umgewandelt und visualisiert.

Schließlich lassen sich Messkosten verringern, indem Leistungsbeiträge und damit die Qualitätssicherung auf Anbieter und Nachfrager aufgeteilt werden. Dies ist Gegenstand einer Externalisierungs- bzw. Internalisierungsstrategie.

\subsubsection{Externalisierung und Internalisierung zur Sen- kung von Messkosten}

Im Rahmen der Dienstleistungserstellung besteht häufig ein gewisser Gestaltungsfreiraum hinsichtlich der Frage, welche Leistung von welchem Transaktionspartner zu erbringen ist. Die Substitution interner gegen externe Produktionsfaktoren bzw. Leistungsbeiträge wird dabei als Externalisierung, die Substitution externer gegen interne Produktionsfaktoren bzw. Leistungsbeiträge als Internalisierung bezeichnet (Corsten 1991; 1997, S. 341).

Aus Sicht der Messkostentheorie entscheidet hierbei der Trade-off zwischen direkten Messkosten und Opportunismuskosten über die Arbeitsteilung. Dies sei am Beispiel einer Pauschalreise verdeutlicht. Grundsätzlich hat der Nachfrager die Möglichkeit, selbst Hotels zu suchen oder die Hotelsuche dem Anbieter zu überlassen. Welche Sorgfalt der Anbieter hierbei aufwendet, stellt für den Nachfrager eine Vertrauenseigenschaft dar. Führt er die Suche selbst durch, so sind die Opportunismuskosten als äußerst gering einzustufen, da man davon ausgehen kann, dass der Nachfrager mit höchster Sorgfalt vorgehen wird; allerdings sind die direkten Messkosten als hoch einzustufen, da er - sofern er nicht bereits in den Hotels übernachtet hat oder über entsprechendes Expertenwissen verfügt - bei der Qualitätsmessung über einen Methodennachteil verfügt. Führt der Reiseveranstalter die Suche durch, so sind die Opportunismuskosten als höher einzustufen, während die Messkosten geringer sind. 
Als Methode für die Entscheidung über Externalisierungs- und Internalisierungsstrategien lässt sich ebenfalls das ServiceBlueprint verwenden. Die Externalisierung entspricht einer Verschiebung der Aktivitäten über die Line of Interaction in den Bereich der Kundenaktivitäten, während die Internalisierung eine Verschiebung über die Line of Interaction in den Anbieterbereich darstellt (Fließ/Völker-Albert 2001).

Externalisierungs- bzw. Internalisierungsstrategien lassen sich mit den verschiedenen Formen der Leistungsbündelung kombinieren. So kann etwa ein Produzent von Speicherchips die Gestaltung des Schaltplanes dem Nachfrager überlassen (Leistungsbündel 1), während er selbst die Herstellung eines Prototyps (Leistungsbündel 2) und die Serienproduktion (Leistungsbündel 3) übernimmt. Die Qualität der jeweiligen Leistungsbündel ist durch entsprechende Messungen (Schaltplan, Prototyp, Serienprodukt) zu prüfen. Da die Qualität des Leistungsergebnisses (Speicherchip in Serie) entscheidend von dem Chipdesign (Schaltplan) abhängt, kann der Nachfrager die Qualität des Leistungsergebnisses durch seine eigenen Leistungsbeiträge steuern. Um die Qualität der Leistungsbeiträge des Nachfragers zu sichern, bedarf es auf Anbieterseite einer Strategie der Qualitätssteuerung.

\subsection{Strategien zur Qualitätssteuerung}

Da Vertrauensgüter durch prohibitiv hohe Messkosten am Output (Leistungsergebnis) gekennzeichnet sind, muss die Qualitätssteuerung am davor liegenden Leistungserstellungsprozess oder sogar am Leistungspotenzial ansetzen (Inputmessung). Von besonderer Bedeutung ist dabei die Abstimmung von internen und externen Faktoren bzw. Leistungsbeiträgen. Hierzu werden sog. Integrationsmittel oder -instrumente (Fließ 1996) eingesetzt. Geht man von der Unterteilung der Einzeltransaktion in die drei Sphären der Information, der Faktorkombination und der Property Rights aus (Kleinaltenkamp 1997, S. 87 ff.), so lassen sich Instrumente mit Bezug zur Faktorkombination (Steuerungsinstrumente) und Instrumente mit Bezug zu den Property Rights (Sicherungsinstrumente) unterscheiden; insgesamt dienen alle Integrationsinstrumente der einseitigen oder zweiseitigen Information der Beteiligten. Steuerungsinstrumente determinieren den weiteren Verlauf des Leistungserstellungsprozesses, in dem sie die Kombination von internen und externen Faktoren steuern. Sicherungsinstrumente beziehen sich auf die Property Rights, d. h. die Verfügungsrechte des Anbieters oder des Nachfragers, sie sichern die Qualität der internen und externen Inputfaktoren und Leistungsbeiträge. Sie schließen vergangene Schritte des Leistungserstellungsprozesses ab und stellen somit Meilensteine für den weiteren Verlauf dar. Einzelinstrumente dienen schwerpunktmäßig entweder der Sicherung oder der Steuerung, während kombinierte Instrumente für beide Zwecke eingesetzt werden können. Tab. 1 gibt einen Überblick über verschiedene Instrumente. Die Instrumente können sowohl zur Steuerung und Sicherung der externen Faktoren und Leistungsbei-

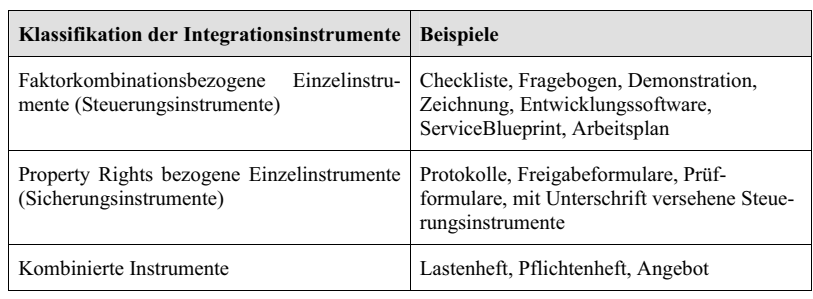

Tab. 1: Integrationsinstrumente zur Steuerung und Sicherung der Qualität im Leistungserstellungsprozess

träge als auch zur Steuerung der internen Faktoren und Leistungsbeiträge eingesetzt werden.

Integrationsinstrumente definieren für den Kunden Messzeitpunkt, Messgegenstand und Messmethode. Sie visualisieren somit Teilaspekte des Leistungserstellungsprozesses und liefern die Grundlage der Qualitätsbeurteilung. Dies ist insbesondere dann relevant, wenn Vertrauenseigenschaften nicht auf der Unzugänglichkeit von Qualitätsmerkmalen beruhen, sondern auf der Uninterpretierbarkeit, etwa aufgrund mangelnden Fachwissens. Steuerungs- und Sicherungsinstrumente sind mit den Messzeitpunkten zu verknüpfen. Sie können im Rahmen des ServiceBlueprints an den Kundenkontaktpunkten auf der Interaktionslinie eingeplant werden.

Neben Integrationsinstrumenten können auch Dienstleistungsstandards zur Qualitätssteuerung herangezogen werden. Unter einem Dienstleistungsstandard soll eine Vorgabe für die Ausgestaltung von Dienstleistungen verstanden werden (Zeithaml/Bitner 2000, S. 224; Kleinaltenkamp 1994, S. 198), die mindestens von einem Hersteller und einem Anwender akzeptiert wird [3]. Dienstleistungsstandards können sich auf alle drei Dimensionen der Leistung beziehen. Standards beinhalten eine Vereinbarung zwischen Anbieter und Nachfrager über den Messzeitpunkt, den Messgegenstand und die Messmethode, insbesondere aber über den Maßstab zur Bewertung von Aktivitäten. Qualitätsstandards können aus den Kundenanforderungen abgeleitet (kundenbezogene Qualitätsstandards), aber auch durch die Realisierungsmöglichkeiten des Anbieters vorgegeben werden (anbieterbezogene Qualitätsstandards). Für den kundigen Nachfrager stellen Qualitätsstandards Möglichkeiten der Qualitätsbewertung dar; verborgene Aktivitäten werden dadurch punktuell sichtbar und nachprüfbar gemacht. Für den unkundigen Nachfrager können Qualitätsstandards als Qualitätsindikatoren gelten.

Qualitätsstandards können im ServiceBlueprint an den verschiedenen Linien etabliert werden: An der Line of Interaction stellen sie für den Kunden nachprüfbare Qualitätsstandards im Hinblick auf bereits erbrachte Leistungen des Anbieters, aber auch im Hinblick auf die Interaktion zwischen Anbieter und Kunde (Interaktionsstandards) dar. Externe Faktor-Standards richten sich auf die vom Kunden zu erfüllenden Qualitätsanforderungen und können die Grundlage für die Marktsegmentierung im Sinne von Self-Selection-Schemes darstellen, z. B. ein bestimmtes Vorwissen, das für die Selbstbedienung not- 
wendig ist. Standards an der Line of Visibility legen fest, was der Kunde sehen sollte und wie die sichtbaren Aktivitäten im Verhältnis zu seinen Anforderungen bewertet werden. An der Line of Internal Interaction beinhalten sie für Anbieter zur Steuerung verwendbare Informationen, die bei Bedarf an den Nachfrager weitergegeben werden können. Im horizontalen Verlauf der Aktivitäten können Standards verschiedene Aktivitätenfolgen abschließen, z. B. durch zeitbezogene Standards.

Qualitätsgestaltung und Qualitätssteuerung sind im Rahmen der Qualitätsentwicklung zeitbezogen zu betrachten. Dies soll im nächsten Abschnitt erfolgen.

\subsection{Qualitätsentwicklung im Zeitablauf}

Prohibitiv hohe Messkosten des Outputs eröffnen opportunistisch zu nutzende Verhaltensspielräume für Anbieter von Vertrauensgütern. Dies gilt für eine einzelne Transaktion, aber auch für eine Vielzahl von Transaktionen im Zeitablauf, d. h. für das Verhalten des Anbieters im Markt. Wie Darby und Karni am Beispiel der Reparaturdienstleistungen gezeigt haben, ist es möglich, bei Unterauslastung der Kapazitäten mehr Dienstleistungen zu verkaufen als aufgrund des Zustands der Pkws, Maschinen etc. tatsächlich benötigt würden (Darby/Karni 1973). Dieses Argument lässt sich auch auf die Qualitätsentwicklung übertragen.

Bei Kapazitätsunterauslastung kann das vom Anbieter angestrebte Qualitätsniveau genau erreicht werden. Aufgrund des Messfehlers ist der Nachfrager allerdings nicht in der Lage, das tatsächliche Qualitätsniveau zu erfassen. Das von ihm wahrgenommene Qualitätsniveau weicht genau um den Messfehler nach oben oder unten vom tatsächlichen Qualitätsniveau ab, d. h. die Qualität wird über- oder unterschätzt. Die oben beschriebenen Maßnahmen der Qualitätsgestaltung und -steuerung ermöglichen eine Qualitätsmessung bzw. -signalisierung und senken somit den Messfehler, d. h. die wahrgenommene Qualität nähert sich der tatsächlichen Qualität an.

Mit zunehmender Auslastung des Anbieterunternehmens und mit zunehmender Annäherung an die Kapazitätsgrenze wird jedoch eine kritische Qualitätsschwelle erreicht. Geht man vereinfachend davon aus, dass die Kapazität in Arbeitsstunden gemessen wird, so steht für jeden Auftrag immer weniger Zeit zur Verfügung, so dass - unterstellt man eine qualitätskritische Zeitmenge pro Auftrag - das Qualitätsniveau sinkt. Aufgrund des Messfehlers sinkt das tatsächliche Messniveau schneller als das vom Nachfrager wahrgenommene Qualitätsniveau. Die Differenz zwischen dem tatsächlichen und dem vom Nachfrager wahrgenommenen Qualitätsniveau ist umso geringer, je geringer der Messfehler ist, d. h. je besser die vom Anbieter durchgeführten Qualitätsstrategien greifen. Um das angestrebte Qualitätsniveau langfristig aufrechtzuerhalten, ist eine Aufstockung der Kapazitäten notwendig. Der optimale Aufstockungszeitpunkt liegt dort, wo die kritische Wahrnehmungsschwelle des Nachfragers überschritten wird. Geht man allerdings davon aus, dass Qualitätsgestaltungs- und -steuerungsmaßnahmen in Verbindung mit der Qualitätsmessung Zeit benötigen, um im Markt bemerkt zu werden, so setzt sich das Qualitätswahrnehmungsgap (Differenz zwischen wahrgenommenem und tatsächlichen Qualitätsniveau) aus einem Messfehlergap und einem Time-lag zusammen.

Der Zusammenhang von Kapazitätsauslastung und tatsächlicher und wahrgenommener Qualität lässt sich auch für Qualitätsentwicklungsstrategien des Up- oder Downgrading benutzen. Der Messfehler, der dem Anbieter einen opportunistischen Verhaltensspielraum eröffnet, gibt den Spielraum für das Downgrading der Qualität vor. Die Qualität kann so lange herunter gefahren werden, bis die aus dem Messfehler und dem Time-lag bestehende Qualitätsdifferenz zwischen tatsächlicher und wahrgenommener Qualität ausgeschöpft ist.

Ein Qualitäts-Upgrading lohnt sich immer dann, wenn dadurch entweder ein höherer Preis durchgesetzt werden kann oder der Anbieter bei gleichem Preis eine höhere Nachfragemenge auf sich ziehen kann. Beim Upgrading ergibt sich die Grenze dort, wo die zusätzlichen Kosten der Qualitätsgestaltung und -steuerung, die die Wahrnehmung höherer Qualität hervorrufen, den zusätzlichen Erlösen, die sich aus einer Preis- und/oder Mengenkomponente zusammensetzen, entsprechen. Da der Messfehler sich sowohl auf positive als auch negative Abweichungen von der tatsächlichen Qualität beziehen kann, kann ein Upgrading auch zu einer höheren wahrgenommenen Qualität führen als es der tatsächlichen entspricht.

\section{Zusammenfassung}

Such-, Erfahrungs- und Vertrauenseigenschaften unterscheiden sich durch die Höhe der Messkosten, wobei Vertrauenseigenschaften und damit auch Vertrauensgüter immer dadurch gekennzeichnet sind, dass die Messkosten des Leistungsergebnisses, d. h. die Messkosten des Outputs, prohibitiv hoch sind. Um sich als qualitätsstarker Anbieter im Wettbewerb profilieren zu können, sind daher Qualitätsstrategien Erfolg versprechend, die die prohibitiv hohen Messkosten des Outputs reduzieren. Hierzu stehen drei Strategien zur Verfügung:

- Die Strategie des Debundling, die in zwei Varianten auftreten kann - als outputbezogenes Debundling, wobei ein Vertrauensgut-Leistungsbündel in mehrere Erfahrungsgut-Einzelkomponenten zerlegt wird, und als prozessbezogenes Debundling, bei dem ein Leistungserstellungsprozess durch verschiedene Messpunkte in Abschnitte zerlegt wird.

- Die Strategie der Visualisierung, die ebenfalls in zwei Varianten auftreten kann - als outputbezogene Visualisierung, bei der Einzelkomponenten eines Vertrauensgutes sichtbar und nachvollziehbar gemacht und somit in Erfahrungskomponenten überführt werden sowie als prozessbezogene Visualisierung, bei der die Line of Visibility verschoben wird. 
- Die Strategie der Internalisierung bzw. Externalisierung, bei der Anbieter und Nachfrager ihre Arbeitsteilung und damit die Messmöglichkeiten variieren.

Als Methoden der Qualitätsgestaltung stehen das House of Quality und das ServiceBlueprint zur Verfügung.

Qualität kann zusätzlich durch eine Strategie der Qualitätssteuerung verdeutlicht werden. Hierzu dienen Integrationsinstrumente in Form von Steuerungs- und Sicherungsinstrumenten sowie Qualitätsstandards.

Die Senkung von Messkosten und die Überführung von Vertrauens- in Erfahrungs- und Sucheigenschaften können nicht vollständig gelingen; ein Messfehler sorgt für eine Abweichung zwischen wahrgenommener und tatsächlicher Qualität. Dieser Messfehler gibt den Verhaltensspielraum für die Strategien des Qualitäts-Upgrading und des Qualitäts-Downgrading sowie eine qualitätsbezogene Wachstumsstrategie von Dienstleistern, die Vertrauensgüter anbieten, vor.

\section{Anmerkungen}

[1] Betrachtet man lediglich das Messfehler-Problem, so weisen die hierfür aufgezeigten Lösungen eine enge Parallele zu den Suchansätzen der Informationsökonomie auf. Betrachtet man das ,cheating”-Problem, so bildet der Messkostenansatz die Basis für die Principal-Agent-Theorie, die sich mit Informations- und Anreizproblemen auseinandersetzt. Hierbei stehen Messprobleme des Verhaltens im Vordergrund.

[2] $\mathrm{Zu}$ abstrahieren ist bei diesem Beispiel von unterschiedlichen Reputationen von Hausarzt und Spezialist, wodurch die Debundlingstrategie konterkariert werden kann, da der Diagnose des Spezialisten mehr vertraut wird als der Diagnose des Hausarztes.

[3] Der Begriff Standard wird in der Literatur unterschiedlich verstanden (siehe zu einem Überblick Kleinaltenkamp 1994, S. 198 ff.)

\section{Literaturverzeichnis}

Adler, J. (1996): Informationsökonomische Fundierung von Austauschprozessen, Wiesbaden.

Akao, Y. (1990): Quality Function Deployment. Integrating Customer Requirements into Product Design, Productivity Press.

Alchian, A.A./Demsetz, H. (1972): Production, Information Costs, and Economic Organization; in: The American Economic Review, Vol. 62, pp. 777-795.

Alchian, A.A./Woodward, S. (1988): The Firm is Dead; Long Live the Firm, in: Journal of Economic Literature, Vol. 26, pp. 65-79.

Arrow, K.J. (1985): The Economics of Agency, in: Pratt, J.W./ Zeckhauser, R.J. (Eds.): Principals and Agents. The Structure of Business, Boston, pp. 37-51.

Barzel, Y. (1982): Measurement Cost and the Organization of Markets, in: Journal of Law and Economics, Vol. 25, pp. 27-48.

Barzel, Y. (1985): Transaction Costs: Are They Just Costs?, in: Journal of Institutional and Theoretical Economics, Vol. 141, pp. 4-16.

Brentani, U. de/Ragot, E. (1996): Developing New Business-toBusiness Professional Services: What Factors Impact Performance?, in: Industrial Marketing Management, Vol. 25, pp. 517530 .

Bruhn, M. (2001): Qualitätsmanagement für Dienstleistungen: Grundlagen, Konzepte, Methoden, 3. Aufl., Berlin u. a.

Bruhn, M./Stauss, B. (Hrsg.) (2000): Dienstleistungsqualität. Konzepte - Methoden - Erfahrungen, 3. Aufl., Wiesbaden.
Cheung, S.N.S. (1969): Transaction Costs, Risk Aversion, and the Choice of Contractual Arrangements, in: The Journal of Law and Economics, Vol. 12, pp. 23-42.

Cheung, S.N.S. (1983): The Contractual Nature of the Firm, in: Journal of Law and Economics, Vol. 26, pp. 1-21.

Corsten, H. (1991): Externalisierung und Internalisierung als strategische Optionen von Dienstleistungsunternehmungen, in: Bruhn, M./Stauss, B. (Hrsg.): Dienstleistungsqualität. Konzepte - Methoden - Erfahrungen, Wiesbaden, S. 165-182.

Corsten, H. (1996): Rationalisierungsmanagement in Dienstleistungsunternehmungen, Arbeitspapier Nr. 6 der Schriften zum Produktionsmanagement der Universität Kaiserslautern, Kaiserslautern.

Corsten, H. (1997): Dienstleistungsmanagement, 3. Aufl., München/Wien.

Darby, M.R./Karni, E. (1973): Free Competition and the Optimal Amount of Fraud, in: Journal of Law and Economics, Vol. 17, pp. 67-88.

Ellinger, T. (1966): Die Informationsfunktion des Produktes, in: Moxter, A./Schneider, D./Wittmann, W. (Hrsg.): Produktionstheorie und Produktionsplanung, Köln/Opladen, S. 253-336.

Emons, W. (2001): Credence Goods Monopolists, in: International Journal of Industrial Organization, Vol. 19, pp. 375-389.

Engelhardt, W.H./Kleinaltenkamp, M./Reckenfelderbäumer, $M$. (1993): Leistungsbündel als Absatzobjekte. Ein Ansatz zur Überwindung der Dichotomie von Sach- und Dienstleistungen, in: Zeitschrift für betriebswirtschaftliche Forschung, 45. Jg., S. 395-426.

Ennew, C.T./Binks, M.R. (1999): Impact of Participative Service Relationship on Quality, Satisfaction and Retention. An Exploratory Study, in: Journal of Business Research, Vol. 46, pp. 121-132.

Eringa, K./Boer, I.L.J. (1998): The Use of Blueprinting and Quality Function Deployment in Restructuring (Educational) Service Processes, in: Quality Management in Services VIII, Proceedings of the EIASM Workshop, Wirtschaftswissenschaftliche Fakultät Ingolstadt der Katholischen Universität Eichstätt, Ingolstadt, pp. 515-525.

Fließ, S. (1996): Prozeßevidenz als Erfolgsfaktor der Kundenintegration, in: Kleinaltenkamp, M./Fließ, S./Jacob, F. (Hrsg.): Customer Integration. Von der Kundenorientierung zur Kundenintegration, Wiesbaden, S. 91-105.

Fließ, S. (2001): Die Steuerung von Kundenintegrationsprozessen. Effizienz in Dienstleistungsunternehmen, Wiesbaden.

Fließ, S./Völker-Albert, J.-H. (2001): Going virtual - Blueprinting als Basis für das Prozessmanagement von E-Service-Anbietern, in: Bruhn, M./Stauss, B. (Hrsg.): E-Services. Dienstleistungsmanagement Jahrbuch 2002, Wiesbaden, S. 263-291.

Gersch, M. (1995): Die Standardisierung integrativ erstellter Leistungen, Arbeitspapier Nr. 57 des Instituts für Unternehmensführung und Unternehmungsforschung der Ruhr-Universität Bochum, Bochum.

Gersch, M. (1998): Vernetzte Geschäftsbeziehungen. Die Nutzung von EDI als Instrument des Geschäftsbeziehungsmanagement, Wiesbaden.

Gössinger, R. (2003): Produktionstheoretische Modellierung des Dienstleistungs-Output der Endkombination, Arbeitspapier Nr. 53 der Schriften zum Produktionsmanagement, Lehrstuhl für Produktionsmanagement, Universität Kaiserslautern, Kaiserslautern.

Gutenberg, E. (1983): Grundlagen der Betriebswirtschaftslehre, Erster Band: Die Produktion, 24. Aufl., Berlin u. a.

Hallwood, C.P. (1990): Measurement Cost and the Organization of Exchange in the Oil Gathering Business, in: Journal of Institutional and Theoretical Economics, Vol. 146, pp. 576-593.

Helm, S. (1997): Relational Uncertainty in Marketing - The Prospect of Integration; in: Lefebvre, E./Cooper, R. (Eds.): Proceedings „Uncertainty, Knowledge and Skill“, Vol. 1, 6.-8. November 1997, Limburg University, Belgium.

Helm, S./Kuhl, M. (1997): Quality Uncertainty and Customer Integration - The Vendor's Perspective; in: Mazet, F./Salle, R./Val- 
la, J.-P. (Eds.): Interaction, Relationships and Networks in Business Markets, Proceedings of the $13^{\text {th }}$ IMP Conference, Lyon, pp. 239-261.

Hempe, S. (1997): Grundlagen des Dienstleistungsmanagements und ihre strategischen Implikationen, Bayreuth.

Hilke, W. (1989): Grundprobleme und Entwicklungstendenzen des Dienstleistungs-Marketing, in: Hilke, W. (Hrsg.): Dienstleistungs-Marketing, Wiesbaden, S. 5-44.

Kaas, K.P. (1990): Marketing als Bewältigung von Informationsund Unsicherheitsproblemen im Markt, in: Die Betriebswirtschaft, 50. Jg., S. 539-548.

Kaas, K.P. (1991): Marktinformationen: Screening und Signaling unter Partnern und Rivalen, in: Zeitschrift für Betriebswirtschaft, 61. Jg., S. 357-370.

Kaas, K.P. (1992a): Kontraktgütermarketing als Kooperation zwischen Prinzipalen und Agenten, in: Zeitschrift für betriebswirtschaftliche Forschung, 44. Jg., S. 884-901.

Kaas, K.P. (1992b): Marketing für umweltfreundliche Produkte, in: Die Betriebswirtschaft, 52. Jg., S. 473-487.

Kaas, K.P./Busch, A. (1996): Inspektions-, Erfahrungs- und Vertrauenseigenschaften von Produkten. Theoretische Konzeption und empirische Validierung, in: Marketing ZFP, Nr. 4, S. 243-252.

Kaldor, N. (1950/51): The Economic Aspects of Advertising, in: Review of Economic Studies, Vol. 18, pp. 1-27.

Kelley, S.W./Skinner, S.J./Donnelly, J.H. (1992): Organizational Socialization of Service Customers, in: Journal of Business Research, Vol. 25, No. 3, pp. 197-214.

Kleinaltenkamp, M. (1992): Investitionsgüter-Marketing aus informationsökonomischer Sicht, in: Zeitschrift für betriebswirtschaftliche Forschung, 44. Jg., S. 809-829.

Kleinaltenkamp, M. (1993): Investitionsgüter-Marketing als Beschaffung externer Faktoren, in: Thelen, E./Mairamhof, G.B. (Hrsg.): Dienstleistungsmarketing, Frankfurt a. M., S. 101119.

Kleinaltenkamp, M. (1994): Technische Standards als Signale im Marktprozess, in: Zahn, E. (Hrsg.): Technologie-Management und Technologien für das Management, Stuttgart, S. 197-226.

Kleinaltenkamp, M. (1997): Integrativität als Kern einer umfassenden Leistungslehre, in: Backhaus, K./Günther, B./Kleinaltenkamp, M./Plinke, W./Raffée, H. (Hrsg.): Marktleistung und Wettbewerb. Strategische und operative Perspektiven der marktorientierten Leistungsgestaltung, Wiesbaden, S. 83-114.

Kleinaltenkamp, M. (2000): Blueprinting? Grundlage des Managements von Dienstleistungsunternehmen, in: Woratschek, H. (Hrsg.): Neue Aspekte des Dienstleistungsmarketing - Ansatzpunkte für Forschung und Praxis, Wiesbaden, S. 3-28.

Kleinaltenkamp, M./Haase, M. (1999): Externe Faktoren in der Theorie der Unternehmung, in: Albach, H./Eymann, E./Luhmer, A./Steven, M. (Hrsg.): Die Theorie der Unternehmung in Forschung und Praxis, Berlin u. a., S. 167-194.

Kleinaltenkamp, M./Marra, A. (1997): Kapazitätsplanung bei Integration externer Faktoren, in: Corsten, H./Stuhlmann, S. (Hrsg.): Kapazitätsmanagement in Dienstleistungsunternehmungen. Grundlagen und Gestaltungsmöglichkeiten, Wiesbaden, S. 55-80.

Krimm, O. (1995): Beitrag zur Produktionsplanung und -steuerung von technischen Dienstleistungen, Dortmund.

Lancaster, K.J. (1966): A New Approach to Consumer Theory, in: Journal of Political Economy, Vol. 74, pp. 132-157.

Malicha, R. (2000): Nachfrageevidenz: Ein Erklärungsansatz zur wahrgenommenen Unsicherheit des Nachfragers bei der Beschaffung von Dienstleistungen?, in: Woratschek, H. (Hrsg.): Neue Aspekte des Dienstleistungsmarketing. Konzepte für Forschung und Praxis, Wiesbaden, S. 131-154.

McManus, J.C. (1975): The Costs of Alternative Economic Organizations, in: Canadian Journal of Economics, Vol. 8, pp. 334-350.

Meffert, H./Bruhn, M. (2003): Dienstleistungsmarketing, 4. Aufl., Wiesbaden.
Meyer, A. (1987): Die Automatisierung und Veredlung von Dienstleistungen - Auswege aus der dienstleistungsinhärenten Produktivitätsschwäche, in: Jahrbuch der Absatz- und Verbrauchsforschung, 33. Jg., S. 25-46.

Meyer, A./Westerbarkey, P. (1995): Bedeutung der Kundenbeteiligung für die Qualitätspolitik von Dienstleistungsunternehmen, in: Bruhn, M./Stauss, B. (Hrsg.): Dienstleistungsqualität: Konzepte Methoden - Erfahrungen, 2. Aufl., Wiesbaden, S. 81-103.

Mills, P.K. (1990): On the Quality of Services in Encounters: An Agency Perspective, in: Journal of Business Research, Vol. 20, pp. $31-41$.

Nelson, P. (1970): Information and Consumer Behavior, in: Journal of Political Economy, Vol. 78, pp. 311-329.

North, D.C. (1990): Institutions, Institutional Change and Economic Performance, Cambridge et al.

North, D.C. (1992): Institutionen, institutioneller Wandel und Wirtschaftsleistungen, Tübingen.

Olderog, T./Skiera, B. (2000): The Benefits of Bundling Strategies, in: SBR/ZfbF, Schmalenbach Business Review/Zeitschrift für betriebswirtschaftliche Forschung, 52. Jg., S. 137-159.

Ouchi, W.G. (1980): Markets, Bureaucracies and Clans, in: Administrative Science Quarterly, Vol. 25, pp. 130-141.

Priemer, V. (2000): Bundling im Marketing. Potenziale - Strategien - Käuferverhalten, Frankfurt a. M. u. a.

Ross, S.A. (1973): The Economic Theory of Agency: The Principal's Problem, in: The American Economic Review, Vol. 63, pp. 134-139.

Saatweber, J. (1994): Quality Function Deployment, in: Masing, W. (Hrsg.): Handbuch Qualitätsmanagement, 3. Aufl., München, S. 445-468.

Schade, C./Schott, E. (1993): Kontraktgüter im Marketing, in: Marketing ZFP, Nr. 1, S. 15-25.

Schneider, B./Bowen, D.E. (1983): New Services Design, Development and Implementation and the Employee, in: George, W.R./Marshall, C.E. (Eds.): Developing New Services, Chicago, pp. 82-101.

Schnittka, M. (1998): Kapazitätsmanagement von Dienstleistungsunternehmungen. Eine Analyse aus Anbieter- und Nachfragersicht, Wiesbaden.

Simon, H. (1994): Preispolitik für industrielle Dienstleistungen, in: Die Betriebswirtschaft, 54. Jg., S. 719-737.

Spence, M.A. (1974): Market Signaling: Informational Transfer in Hiring and Related Screening Processes, Cambridge Mass.

Spremann, K. (1988): Reputation, Garantie, Information, in: Zeitschrift für Betriebswirtschaft, 58. Jg., S. 613-629.

Stuart, F.I./Tax, S.S. (1996): Planning for Service Quality: An Integrative Approach, in: International Journal of Service Industry Management, Vol. 7, pp. 58-77.

Weiber, R./Adler, J. (1995a): Informationsökonomisch begründete Typologisierung von Kaufprozessen, in: Zeitschrift für betriebswirtschaftliche Forschung, 47. Jg., S. 43-65.

Weiber, R./Adler, J. (1995b): Positionierung von Kaufprozessen im informationsökonomischen Dreieck: I Operationalisierung und verhaltenswissenschaftliche Prüfung, in: Zeitschrift für betriebswirtschaftliche Forschung, 47. Jg., S. 99-123.

Weiber, R./Jacob, F. (2000): Kundenbezogene Informationsgewinnung; in: Kleinaltenkamp, M./Plinke, W. (Hrsg.): Technischer Vertrieb - Grundlagen des Business-to-Business-Marketing, 2. Aufl., Berlin, S. 523-612.

Weißenberger, B. (1998): Zur Bedeutung von Vertrauensstrategien für den Aufbau und Erhalt von Kundenbindung im Konsumgüterbereich, in: Zeitschrift für betriebswirtschaftliche Forschung, 50. Jg., S. 614-640.

Williamson, O.E. (1996): The Mechanisms of Governance, New York.

Witte, E. (1973): Organisation für Innovationsentscheidungen, Göttingen.

Wolinsky, A. (1995): Competition in Markts for Credence Goods, in: Journal of Institutional and Theoretical Economics, Vol. 151, pp. 117-131. 
Woratschek, H. (1996): Die Typologie von Dienstleistungen aus informationsökonomischer Sicht, in: der markt, 35. Jg., Nr. 136, S. 59-71.

Yadav, M.S./Monroe, K.B. (1993): How Buyers Perceive Savings in a Bundle Price: An Examination of a Bundle's Transaction Value, in: Journal of Marketing Research, Vol. 30, pp. 350-358.

Zeithaml, V./Bitner, M.J. (2000): Services Marketing. Integrating the Customer Focus Across the Firm, $2^{\text {nd }}$ ed., New York et al.

\section{Summary}

Credence qualities refer to characteristics of goods which are expensive to judge even after purchase. They typically arise with services, especially complex services like consulting. As credence qualities cannot easily be evaluated by customers, credence qualities also raise certain problems for suppliers in planning, managing and improving quality. Based on the measurement cost approach of transaction cost theory, the article discusses different quality strategies for suppliers like debundling, visualization and internalization / externalization of service components and parts of the service process. It further suggests different instruments and tools suppliers can use to control and improve quality with credence goods. It closes with a discussion of the limits of quality up- and downgrading.

\section{Schlüsselbegriffe}

Vertrauensgüter, Qualitätsmanagement, Debundlingstrategie, Internalisierung, Externalisierung, Quality Function Deployment

\section{Keywords}

Credence goods, service quality management, debundling, quality function deployment, service blueprint

\section{MARKETING}

Schriftleitung: Verantwortlich: Univ.-Prof. Dr. Bernd Stauss, Lehrstuhl für ABWL und Dienstleistungsmanagement, Katholische Universität Eichstätt-Ingolstadt, WFI, Auf der Schanz 49, 85049 Ingolstadt, Tel. 0841/937-1861, Fax 0841/937-1970, marketing-zfp@dlm-stauss.de

Herausgeber: Univ.-Prof. Dr. Hans H. Bauer, Mannheim Univ.-Prof. Dr. Hermann Diller, Erlangen-Nürnberg, Univ.-Prof. Dr. Hans Mühlbacher, Innsbruck, Univ.-Prof Dr. Bernd Stauss, Eichstätt, Univ.-Prof. Dr. Peter Weinberg, Saarbrücken, Univ.-Prof. Dr. Joachim Zentes, Saarbrücken.

Mit der Annahme eines Manuskripts zur Veröffentlichung überträgt der Autor dem Verlag das ausschließliche Verlagsrecht für die Zeit bis zum Ablauf des Urheberrechts. Eingeschlossen sind insbesondere auch die Befugnis zur Einspeicherung in eine Datenbank sowie das Rech der weiteren Vervielfältigung zu gewerblichen Zwecken im Wege eines photomechanischen oder eines anderen Verfahrens. Dem Autor verbleibt die Befugnis, nach Ablauf eines Jahres anderen Verlagen eine einfache Abdruck- genehmigung zu erteilen; ein Honorar hieraus steht dem Autor zu.

Urheber- und Verlagsrechte: Alle in dieser Zeitschrift veröffentlichten Beiträge sind urheberrechtlich geschützt. Das gilt auch für die veröffentlichten Gerichtsentscheidungen und ihre Leitsätze, denn diese sind geschützt, sowei sie vom Einsender oder von der Schriftleitung erarbeite oder redigiert worden sind. Der Rechtsschutz gilt auch gegenüber Datenbanken und ähnlichen Einrichtungen. Kei Teil dieser Zeitschrift darf außerhalb der engen Grenzen des Urheberrechtsgesetzes ohne schriftliche Genehmigung des Verlags in irgendeiner Form - durch Fotokopie, Mikrofilm oder andere Verfahren - reproduziert oder in eine von Maschinen, insbesondere von Datenverarbeitungsanlagen verwendbare Sprache, übertragen werden.

Anzeigenabteilung: Verlag C.H. Beck, Anzeigenabteilung, Wilhelmstr. 9, 80801 München. Postanschrift: Postfach 4003 40, 80703 München.

Media-Service: Telefon 089/3 81 89-781, Telefax 089/ 381 89-782,media-service@beck.de

Media-Disposition (Herstellung Anzeigen, technische Daten), Telefon 089381 89-598/-603, Telefax Auftragsservice 089/3 $8189-589$

Verantwortlich für den Anzeigenteil: Fritz Lebherz. Z.Zt. gilt Anzeigenpreisliste Nr. 21. 